\title{
COMBINATORIAL NEGATIVE CURVATURE AND TRIANGULATIONS OF THREE-MANIFOLDS
}

\author{
DAMIAN OSAJDA
}

\begin{abstract}
We introduce and study local combinatorial conditions on a simplicial complex, implying Gromov hyperbolicity of its universal cover. We apply the theory to Thurston's problem on $5 / 6^{*}$-triangulations of 3-manifolds, providing a new proof and generalizing the original result. We indicate further applications.
\end{abstract}

\section{INTRODUCTION}

A $5 / 6^{*}$-triangulation of a 3 -manifold is a triangulation in which every edge has degree 5 or 6 (i.e. there are 5 or 6 tetrahedra around each edge), and every triangle contains only one edge of degree 5 . Thurston conjectured that every closed 3-manifold that admits a 5/6*-triangulation has Gromov hyperbolic fundamental group. Elder, McCammond and Meier EMM03 established this statement, via a computer-assisted proof.

In this paper we place the question on hyperbolic triangulations of 3-manifolds into the frame of a combinatorial nonpositive curvature. We introduce a new local combinatorial condition of $m$-location (where $m=7,8,9, \ldots)$ for flag simplicial complexes. When $m \geqslant 8$ this condition is an analogue of the negative curvature, as the following main result of the paper shows.

Theorem A. Let $X$ be a simply connected 8-located locally 5-large simplicial complex. Then the 1-skeleton of $X$, equipped with the standard path metric, is Gromov hyperbolic.

There are many 8-located simplicial complexes and groups acting on them geometrically resulting from various notions of combinatorial nonpositive curvature appearing in the literature (see Section 5 for a discussion). However, with the notion of $m$-location we enter for the first time the world of triangulations of manifolds in dimensions above 2.

Theorem B. Every 5/6*-triangulation of a 3-manifold is an 8-located locally 5-large simplicial complex.

This provides a new proof of Thurston's conjecture. Our methods are quite elementary and we do not use computer computations. Moreover, Theorem B shows that Theorem A is a generalization of the original statement. There are 8 -located triangulations of 3 -manifolds that are not $5 / 6^{*}$-triangulations. Furthermore, our proof provides a uniform bound on the hyperbolicity constant and describes the structure of combinatorial balls in $5 / 6^{*}$-triangulations.

Clearly, Theorem A applies in a much broader context than the one of 3-manifolds. It concerns general simplicial complexes. In Section 5 we indicate other applications. We also relate 8-location to the well known notion of "combinatorial nonpositive curvature" - systolicity. Note that systolic complexes are in a sense very (asymptotically) far from triangulations of manifolds above dimension 2. This makes the 8-location very interesting, since it may be applied, unlike systolicity, to some classical spaces and groups. In the same Section 5 we briefly discuss $m$-location, for $m<8$, as an analogue of a nonpositive, but not negative, curvature.

The proof of Theorem A is presented in Section 3. It uses a local-to-global technique developed by the author in Osa13. The main global property of simply connected 8-located complexes - the property SD' - is a variation of the main feature of weakly systolic complexes - the simple descent property. In Section 4 we prove Theorem B.

Acknowledgment. I thank Tadeusz Januszkiewicz and Misha Kapovich for discussions on triangulations of manifolds.

Date: June 10, 2015.

2010 Mathematics Subject Classification. 20F67; 57M50.

Key words and phrases. combinatorial nonpositive curvature, three manifold, hyperbolic group. 
This research was supported by Narodowe Centrum Nauki, decision no DEC-2012/06/A/ST1/00259, and by the ERC grant ANALYTIC no. 259527.

\section{Preliminaries}

2.1. Simplicial complexes. Let $X$ be a simplicial complex. The $i$-skeleton of $X$ is denoted by $X^{(i)}$. A subcomplex $Y$ of $X$ is full if every subset $A$ of vertices of $Y$ contained in a simplex of $X$, is contained in a simplex of $Y$. For a finite set $A=\left\{v_{1}, \ldots, v_{k}\right\}$ of vertices of $X$, by $\langle A\rangle$ or by $\left\langle v_{1}, \ldots, v_{k}\right\rangle$ we denote the span of $A$, i.e. the smallest full subcomplex of $X$ containing $A$. Thus " $\langle A\rangle \in X$ " or " $\left\langle v_{1}, v_{2}, \ldots\right\rangle \in X$ " mean that the corresponding sets span a simplex in $X$. We write $v \sim v^{\prime}$ (respectively, $v \nsim v^{\prime}$ ) if $\left\langle v, v^{\prime}\right\rangle \in X$ (respectively, $\left\langle v, v^{\prime}\right\rangle \notin X$ ). Moreover, we write $v \sim v_{1}, v_{2}, \ldots$ (respectively, $v \nsim v_{1}, v_{2}, \ldots$ ) when $v \sim v_{i}$ (respectively, $v \nsim v_{i}$ ) for $i=1,2, \ldots$. A simplicial complex $X$ is flag whenever every finite set of vertices of $X$ joined pairwise by edges in $X$, is contained in a simplex of $X$. A link of a simplex $\sigma$ of $X$ is a simplicial complex $X_{\sigma}=\{\tau \mid \tau \in X \& \tau \cap \sigma=\emptyset \&\langle\tau \cup \sigma\rangle \in X\}$.

Let $k \geqslant 4$. A $k$-cycle $\left(v_{1}, \ldots, v_{k}\right)$ is a triangulation of a circle consisting of $k$ vertices: $v_{1}, \ldots, v_{k}$, and $k$ edges: $\left\langle v_{i}, v_{i+1}\right\rangle$ and $\left\langle v_{k}, v_{1}\right\rangle$. A $k$-wheel (in $\left.X\right)\left(v_{0} ; v_{1}, \ldots, v_{k}\right)$ (where $v_{i}$ 's are vertices of $X$ ) is a subcomplex of $X$ such that $\left(v_{1}, \ldots, v_{k}\right)$ is a full cycle, and $v_{0} \sim v_{1}, \ldots, v_{k}$. A flag simplicial complex $X$ is $k$-large if there are no full $j$-cycles in $X$, for $j<k$. $X$ is locally $k$-large if all its links are $k$-large. Observe that local 5 -largeness means that there are no 4 -wheels.

If not stated otherwise, speaking about a simplicial complex $X$, we always consider the metric on the 0 -skeleton $X^{(0)}$, defined as the number of edges in the shortest 1 -skeleton path joining two given vertices. We denote this metric by $d(\cdot, \cdot)$. Given a nonnegative integer $i$ and a vertex $v \in X$, a (combinatorial) ball $B_{i}(v, X)$ (respectively, sphere $S_{i}(v, X)$ ) of radius $i$ around $v$ (or $i$-ball around $v$ ) is a full subcomplex of $X$ spanned by vertices at distance at most $i$ (respectively, at distance $i$ ) from $v$. We use the notation $B_{i}(v)$ and $S_{i}(v)$ for, respectively, $B_{i}(v, X)$ and $S_{i}(v, X)$, when it is clear what is the underlying complex $X$.
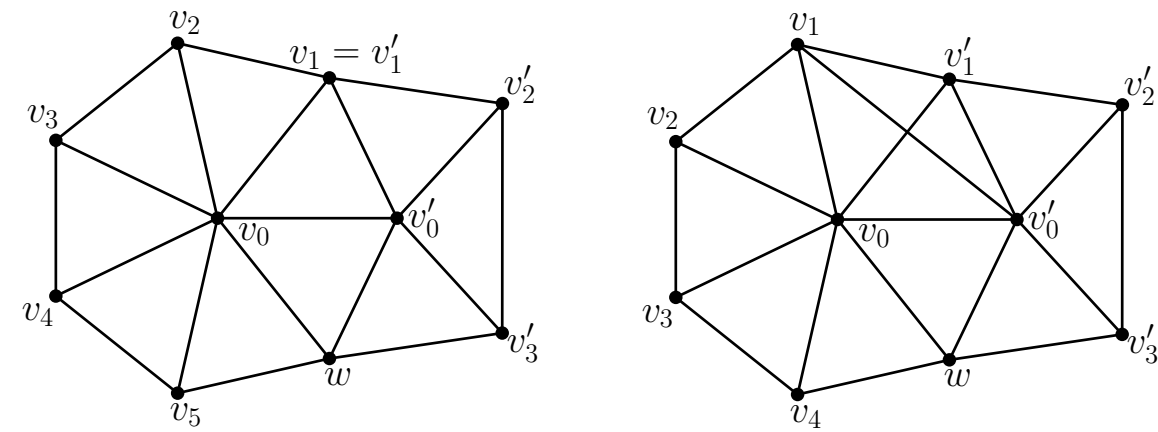

Figure 1. Two types of dwheels with the boundary length 8: a $(7,5)$-dwheel (left), and a $(6,5)$-dwheel (right).

2.2. Location. A $(k, l)$-dwheel (from "double wheel") $d W=\left(v_{0}, v_{0}^{\prime}, w ; v_{1}, \ldots, v_{k-2} ; v_{1}^{\prime}, \ldots, v_{l-2}^{\prime}\right)$ is the union of a $k$-wheel $\left(v_{0} ; v_{1}, \ldots, v_{k-2}, w, v_{0}^{\prime}\right)$ and an $l$-wheel $\left(v_{0}^{\prime} ; v_{1}^{\prime}, \ldots, v_{l-2}^{\prime}, w, v_{0}\right)$, with $v_{1}=v_{1}^{\prime}$ or $v_{1} \sim v_{1}^{\prime}-$ see Figure 1. The boundary of the dwheel $d W$ is the cycle $\left(v_{1}, \ldots, v_{k-2}, w, v_{l-2}^{\prime}, \ldots, v_{1}^{\prime}\right)$, and the boundary length is $k+l-4$ if $v_{1}=v_{1}^{\prime}$ or $k+l-3$ otherwise.

Definition 2.1 ( $m$-location). A flag simplicial complex is $m$-located if every dwheel with the boundary length at most $m$ is contained in a 1-ball - see Figure 2

In this article by a covering we mean a simplicial covering, that is a simplicial map restricting to isomorphisms from 1-balls onto spans of their images. In particular, it follows that wheels are mapped isomorphically onto wheels, and thus the following result holds.

Proposition 2.1. A covering of a locally $k$-large complex is itself locally $k$-large. A covering of an $m$-located complex is itself $m$-located. 

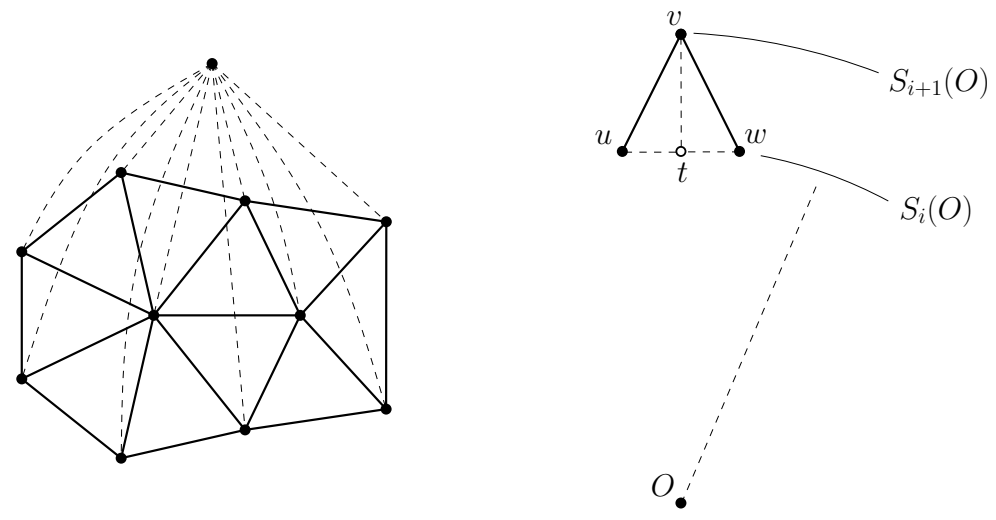

FIGURE 2. 8-location (left), and the vertex condition (V) for the property SD' (right).

\section{Hyperbolicity}

The goal of this section is to prove Theorem A. In Subsection 3.1 we introduce a global combinatorial property $S D^{\prime}$ that, together with 8-location and local 5-largeness, implies hyperbolicity - Theorem 3.3 . In the subsequent Subsection 3.2, we prove a local-to-global Theorem 3.4 implying directly Theorem A.

\subsection{Global condition.}

Definition 3.1 (Property $S D^{\prime}$ ). Let $X$ be a flag simplicial complex, let $O$ be its vertex, and let $n \in$ $\{0,1,2,3, \ldots\}$. We say that $X$ satisfies the property $S D^{\prime}{ }_{n}(O)$ if for every $i=1,2, \ldots, n$ the following two conditions hold.

(T) (Triangle condition): For every edge $e \in S_{i+1}(O)$ the intersection $X_{e} \cap B_{i}(O)$ is non-empty.

(V) (Vertex condition): For every vertex $v \in S_{i+1}(O)$, and for every two vertices $u, w \in X_{v} \cap B_{i}(O)$, there exists a vertex $t \in X_{v} \cap B_{i}(O)$ such that $t \sim u, w$ - see Figure 2

$X$ satisfies the property $S D^{\prime}(O)$ (respectively, the property $S D^{\prime}$ ) if $S D_{n}^{\prime}(O)$ holds for every $n$ (respectively, for every $n$ and $O)$.

Proposition 3.1 (Simple connectedness). Let $X$ be a simplicial complex satisfying the property $S D^{\prime}(O)$, for some vertex $O$. Then $X$ is simply connected.

Proof. The proof follows closely the proof of $\mathrm{BCC}^{+} 13$ Lemma 5.5]. Here, instead of the quadrangle condition one uses the vertex condition (V) from Definition 2.1.

Lemma 3.2. Let $X$ be an 8-located locally 5-large simplicial complex satisfying the property $S D_{n}^{\prime}(O)$, for some vertex $O$. Let $v \in S_{n+1}(O)$ and let $y, z \in X_{v} \cap B_{n}(O)$ be such that $y \nsim z$. Let $x \in X_{v} \cap B_{n}(O)$ be a vertex adjacent to $y, z$, given by the vertex condition $(V)$. Let $y^{\prime}, z^{\prime} \in B_{n-1}(O)$ be vertices with $\left\langle x, y, y^{\prime}\right\rangle,\left\langle x, z, z^{\prime}\right\rangle \in$ $X$, given by the triangle condition $(T)$. Then $y^{\prime} \neq z^{\prime} ; y^{\prime} \nsim z ; y \nsim z^{\prime}$; and $y^{\prime} \sim z^{\prime}$.

Proof. By 5-largeness we have immediately that $y^{\prime} \neq z^{\prime} ; y^{\prime} \nsim z$; and $y \nsim z^{\prime}$. For the rest we proceed by contradiction. Assume that $y^{\prime} \nsim z^{\prime}$. Then, by the vertex condition $(\mathrm{V})$, there is a vertex $x^{\prime} \in S_{n-1}(O)$ adjacent to $x, y, z$. Note that, by 5 -largeness, it is not possible that $y \sim x^{\prime} \sim z$, thus there is a 6 -wheel $\left(x ; v, z, z^{\prime}, x^{\prime}, y^{\prime}, y\right)$, or a 5 -wheel $\left(x ; v, z, z^{\prime}, x^{\prime}, y\right)$, or $\left(x ; v, z, x^{\prime}, y^{\prime}, y\right)$. By the triangle (T) and the vertex $(\mathrm{V})$ condition there are vertices $y^{\prime \prime}, x^{\prime \prime}, z^{\prime \prime} \in S_{n-2}(O)$ with $\left\langle x^{\prime}, y^{\prime}, y^{\prime \prime}\right\rangle,\left\langle x^{\prime}, z^{\prime}, z^{\prime \prime}\right\rangle \in X$, and $x^{\prime \prime} \sim x^{\prime}, y^{\prime \prime}, z^{\prime \prime}$. Possibly $x^{\prime \prime}=z^{\prime \prime}$, but $x^{\prime \prime} \neq y^{\prime \prime}$, by 5 -largeness. In any case we obtain an $(k, l)$-dwheel $d W$, spanned by vertices in $\left\{v, x, y, z, x^{\prime}, y^{\prime}, z^{\prime}, x^{\prime \prime}, y^{\prime \prime}, z^{\prime \prime}\right\}$, with the boundary length at most 8 . Thus, by 8-location, $d W$ is contained in a 1-ball, which contradicts the fact that $d\left(v, y^{\prime \prime}\right)=3$.

Theorem 3.3 (Hyperbolicity). Let $X$ be an 8-located locally 5-large simplicial complex satisfying the property $S D^{\prime}$. Then $X^{(0)}$ equipped with a path metric induced from $X^{(1)}$ is $\delta$-hyperbolic, for a universal constant $\delta$.

Proof. We use a criterion by Papasoglu Pap95], i.e. we reduce the proof to showing that intervals are uniformly thin. Let $O, O^{\prime}$ be two vertices and let $I$ be the interval between them, i.e. the set of vertices lying on geodesics between $O$ and $O^{\prime}$. By $I_{k}$ we denote the intersection $S_{k}(O) \cap I=S_{k}(O) \cap S_{n-k}\left(O^{\prime}\right)$, where 
$n=d\left(O, O^{\prime}\right)$. We show that for every $k \leqslant n$, for every two vertices $v, w \in I_{k}$, we have $d(v, w) \leqslant 2$. This shows also that the hyperbolicity constant is universal.

By contradiction - let $k$ be the maximal number such that there are vertices $v, w \in I_{k}$ with $d(v, w)>2$. Then in $I_{k+1}$ there exist vertices $v^{\prime} \sim v$, and $w^{\prime} \sim w$ with $d\left(v^{\prime}, w^{\prime}\right) \leqslant 2$. By the vertex condition $(\mathrm{V})$, there is a vertex $z \sim v^{\prime}, w^{\prime}$ in $I_{k+1}$, possibly with $z=w^{\prime}$. By the triangle condition (T) there are vertices $v^{\prime \prime}, w^{\prime \prime} \in I_{k}(O)$, with $v^{\prime \prime}=w^{\prime \prime}$ if $z=w^{\prime}$, such that $v^{\prime \prime} \sim v^{\prime}, z$, and $w^{\prime \prime} \sim z, w^{\prime}$. By the vertex condition (V), in $I_{k}$ there exist vertices $s \sim v, v^{\prime}, v^{\prime \prime} ; t \sim v^{\prime \prime}, z, w^{\prime \prime} ; u \sim w, w^{\prime}, w^{\prime \prime}$, possibly, with $s=v^{\prime \prime}, t=w^{\prime \prime}, u=w$. Among the vertices $t, w^{\prime \prime}, u, w$ we choose the first one (in the given order), that is at distance 3 from $v$. Denote this vertex by $v^{\prime \prime \prime}$. Using the vertex condition $(\mathrm{V})$, we obtain a full path $v_{1} v_{2} v_{3} v_{4}$ in $I_{k}$ of diameter 3 , with $v_{1}=v$ and $v_{4}=v^{\prime \prime \prime}$. In the remaining part of the proof we show that the existence of such path is impossible. This leads to a contradiction yielding the theorem.

For $i=1,2,3$, let $w_{i} \sim v_{i}, v_{i+1}$ be a vertex in $I_{k-1}$ given by the triangle condition $(\mathrm{T})$. All the possible cases (up to renaming vertices) of mutual relations between vertices $w_{i}$ are shown in Figure 3 - Cases: I, II, and III.

Case I. In this case we assume that for all triples of vertices $w_{i}$ as above we are not in Case II or III, that is $w_{i} \neq w_{j}$, for $i \neq j$. First, suppose that $w_{1} \sim w_{2} \sim w_{3}$. We may assume that $w_{1} \nsim w_{3}$. By (T), there are vertices $u_{1}, u_{2} \in I_{k-2}$ with $u_{j} \sim w_{j}, w_{j+1}$. If $u_{1}=u_{2}$ then we are in Case I(a) (see Figure 3p. Further we assume that there are no such $u_{i}$. If $u_{1} \sim u_{2}$ then, by (T), there is a vertex $t \in I_{k-3}$ adjacent to $u_{1}, u_{2}$ - Case I(b) (see Figure 3). If not then, by (V), there is a vertex $u^{\prime} \in I_{k-2}$ adjacent to $u_{1}, u_{2}$. If $u^{\prime} \sim w_{1}$ then we are in the previous case taking $u^{\prime}$ instead of $u_{1}$. Similarly for $u^{\prime} \sim w_{3}$, thus further we assume that $u^{\prime} \nsim w_{1}, w_{3}$. By (T), in $I_{k-3}$ there exist $t_{1}, t_{2}$ with $t_{i} \sim u^{\prime}, u_{i}$. By Lemma 3.2 , we have $t_{1} \neq t_{2}$ and $t_{1} \sim t_{2}$. It follows that there is a $(7,5)$-dwheel $\left(w_{2}, u^{\prime}, u_{2} ; u_{1}, w_{1}, v_{2}, v_{3}, w_{3} ; u_{1}, t_{1}, t_{2}\right) d W$. By 8-location, $d W$ is contained in a 1 -ball - contradiction, since $d\left(v_{2}, t_{1}\right)=3$.
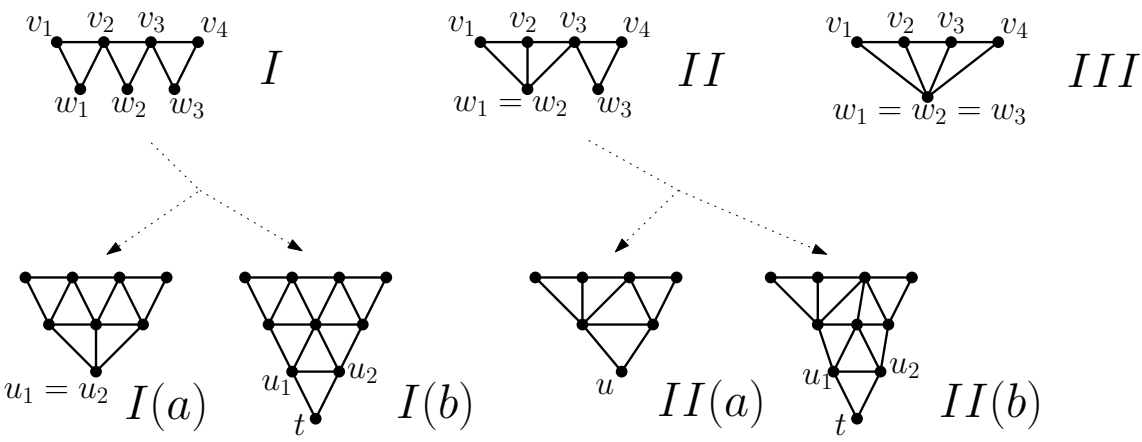

Figure 3. Possible cases in the proof of Theorem 3.3 .

Now we suppose that $w_{1} \sim w_{2}$, and $w_{2} \nsim w_{3}$. Then, by $(\mathrm{V})$, in $I_{k-1}$ there is $w^{\prime} \sim v_{3}, w_{2}, w_{3}$. We may assume that $w^{\prime} \nsim w_{1}$. By (T), in $I_{k-2}$ there are $u_{2}, u_{3}$, with $u_{i} \sim w^{\prime}, w_{i}$. By Lemma $3.2, u_{2} \neq u_{3} ; u_{2} \nsim w_{3}$; $w_{2} \nsim u_{3}$; and $u_{2} \sim u_{3}$. Therefore, $w_{1}, v_{2}, v_{3}, w_{3}, u_{3}$ are contained in a $(5, m)$-dwheel $d W$, with the boundary length at most 8 . By 8 -location, $d W$ is contained in a 1-ball $B_{1}\left(w^{\prime \prime}\right)$. Replacing $w_{2}$ by $w^{\prime \prime}$ we are in Case $\mathrm{I}(\mathrm{a})$ or $\mathrm{I}(\mathrm{b})$.

If $w_{1} \nsim w_{2} \nsim w_{3}$ then, by (V), in $I_{k-1}$ there are $w_{1}^{\prime}, w_{2}^{\prime}$ with $w_{j}^{\prime} \sim v_{j+1}, w_{j}, w_{j+1}$. By $(\mathrm{T})$, in $I_{k-2}$ there exist $u_{1}, u_{1}^{\prime}, u_{2}, u_{2}^{\prime}$ with $u_{j} \sim w_{j}, w_{j}^{\prime}$, and $u_{j}^{\prime} \sim w_{j}^{\prime}, w_{j+1}$. By Lemma $3.2 u_{1} \neq u_{1}^{\prime} ; u_{1} \nsim w_{2} ; w_{1} \nsim u_{1}^{\prime}$; and $u_{1} \sim u_{1}^{\prime}$. Thus the vertices $u_{1}, w_{1}, v_{2}, v_{3}, w_{2}^{\prime}, u_{2}$ are contained in a $(5, m)$-dwheel $d W$, with the boundary length at most 8 . By 8 -location, $d W$ is contained in $B_{1}(w)$, and hence, replacing $w_{2}$ by $w$, we are in the previous case.

Case II. Proceeding similarly as in Case I above (i.e. using the conditions (V) and (T), and Lemma 3.2), we come to the two possible cases: II(a) and II(b), as in Figure 3 - with $u, u_{1}, u_{2} \in I_{k-2}$ and $t \in I_{k-3}$.

In the remaining part of the proof we analyze separately all the cases: I(a), I(b), II(a), II(b), and III, showing that they lead to contradiction. To do this we "project" edges and vertices towards $O^{\prime}$ now: By (T), in $I_{k+1}$ there exist vertices $p_{1}, p_{2}, p_{3}$ (possibly some of them coinciding), with $p_{j} \sim v_{j}, v_{j+1}$. By (V), we have that $d\left(p_{j}, p_{j+1}\right) \leqslant 2$. 
Ad Case III: This leads to a contradiction, since $d\left(v_{1}, v_{4}\right)=3$.

Ad Case II (a): By Lemma 3.2, we have $p_{1} \nsim v_{3} ; v_{1} \nsim p_{2} ; p_{1} \neq p_{2} ;$ and $p_{1} \sim p_{2}$. Therefore, the vertices $p_{1}, v_{1}, w_{1}, w_{3}, v_{4}, p_{3}$ are then contained in a $(5, m)$-dwheel $d W$ with the boundary length at most 8 . Thus, by 8-location, $d W$ is contained in a 1 -ball - contradiction, since $d\left(v_{1}, v_{4}\right)=3$.

Ad Case II(b): By Lemma 3.2 we have $p_{1} \nsim v_{3} ; v_{1} \nsim p_{2} ; p_{1} \neq p_{2}$; and $p_{1} \sim p_{2}$. By $(\mathrm{V})$, there is $p^{\prime} \in I_{k+1}$ with $p^{\prime} \sim p_{2}, p_{3}, v_{3}$, possibly with $p^{\prime}=p_{3}$. We may assume that $p^{\prime} \nsim p_{1}$. By $(\mathrm{T})$, in $I_{k+2}$ there are vertices $q_{1} \sim p_{1}, p_{2}$ and $q_{2} \sim p_{2}, p^{\prime}$, with $d\left(q_{1}, q_{2}\right) \leqslant 2$, by $(\mathrm{V})$. Therefore the vertices $q_{1}, p_{1}, v_{1}, w_{1}, v_{3}, p^{\prime}, q_{2}$ are contained in a $(5, m)$-dwheel $d W$ with the boundary length at most 8 . Thus, by 8 -location, $d W$ is contained in a 1-ball - contradiction, since $d\left(w_{1}, q_{1}\right)=3$.

Ad Case I $(a)$ : The vertices $p_{2}, v_{2}, w_{1}, u_{1}, w_{3}, v_{4}, p_{3}$ are contained in a $(5, m)$-dwheel $d W$ with the boundary length at most 8 . Thus, by 8 -location, $d W$ is contained in a 1 -ball - contradiction, since $d\left(u_{1}, p_{3}\right)=3$.

Ad Case I(b): If $p_{1}=p_{2}$ or $p_{1} \sim p_{2}$ then we obtain a $(6, m)$-dwheel containing $p_{1}, v_{1}, w_{1}, u_{1}, u_{2}, w_{3}, v_{3}, p_{2}$, leading to contradiction, by 8-location. Similarly, when $p_{2}=p_{3}$ or $p_{2} \sim p_{3}$. Thus, further we assume that $d\left(p_{j}, p_{j+1}\right)=2$. By $(\mathrm{V})$, in $I_{k+1}$ there is a vertex $p^{\prime} \sim p_{1}, p_{2}$. We may assume that $v_{1} \nsim p^{\prime} \nsim v_{3}$. By (T), in $I_{k+2}$ there exist vertices $q_{1}, q_{2}$, such that $q_{j} \sim p_{j}, p^{\prime}$. By Lemma 3.2, $q_{1} \neq q_{2} ; q_{1} \nsim p_{2} ; p_{1} \nsim q_{2} ;$ and $q_{1} \sim q_{2}$. Thus there is a $(7,5)$-dwheel $\left(v_{2}, p^{\prime}, p_{2} ; p_{1}, v_{1}, w_{1}, w_{2}, v_{3} ; p_{1}, q_{1}, q_{2}\right)$, which lies in a 1 -ball, by 8 -location. This however contradicts the fact that $d\left(w_{1}, q_{1}\right)=3$.

3.2. Local-to-global. In this section we prove the following result implying Theorem A from Introduction.

Theorem 3.4 (Local-to-global). Let $X$ be an 8-located locally 5-large simplicial complex. Then its universal cover $\widetilde{X}$ is an 8-located locally 5-large simplicial complex satisfying the property $S D^{\prime}$. In particular, $\widetilde{X}^{(1)}$ is $\delta$-hyperbolic.

Proof. The proof follows closely - up to much of the notations - the proof of the analogous Theorem 4.5 from Osa13 (compare also the proof of $\mathrm{BCC}^{+} 13$. Theorem 1]). We construct the universal cover $\widetilde{X}$ of $X$ as an increasing union $\bigcup_{i=1}^{\infty} \widetilde{B}_{i}$ of combinatorial balls. The covering map is then the union

$$
\bigcup_{i=1}^{\infty} f_{i}: \bigcup_{i=1}^{\infty} \widetilde{B}_{i} \rightarrow X
$$

where $f_{i}: \widetilde{B}_{i} \rightarrow X$ is locally injective and $\left.f_{i}\right|_{\widetilde{B}_{j}}=f_{j}$, for $j \leqslant i$.

We proceed by induction. Choose a vertex $O$ of $X$. Define $\widetilde{B}_{0}=\{O\}, \widetilde{B}_{1}=B_{1}(O, X)$ and $f_{1}=\operatorname{Id}_{B_{1}(O)}$. Assume that we have constructed the balls $\widetilde{B}_{1}, \widetilde{B}_{2}, \ldots, \widetilde{B}_{i}$ and the corresponding maps $f_{1}, f_{2}, \ldots, f_{i}$ to $X$ so that the following conditions are satisfied:

$\left(P_{i}\right): \widetilde{B}_{j}=B_{j}\left(O, \widetilde{B}_{i}\right)$ for $j=1,2, \ldots, i$;

$\left(Q_{i}\right): \widetilde{B}_{i}$ satisfies the property $S D_{i-1}^{\prime}(O)$

$\left(R_{i}\right):\left.f_{i}\right|_{B_{1}\left(\widetilde{w}, \widetilde{B}_{i}\right)}: B_{1}\left(\widetilde{w}, \widetilde{B}_{i}\right) \rightarrow B_{1}\left(f_{i}(\widetilde{w}), X\right)$ is an isomorphism onto the span of the image for $\widetilde{w} \in \widetilde{B}_{i}$ and it is an isomorphism for $\widetilde{w} \in \widetilde{B}_{i-1}$.

Observe that those conditions are satisfied for $\widetilde{B}_{1}$ and $f_{1}$, i.e. that conditions $\left(P_{1}\right),\left(Q_{1}\right)$ and $\left(R_{1}\right)$ hold. Now we construct $\widetilde{B}_{i+1}$ and the map $f_{i+1}: \widetilde{B}_{i+1} \rightarrow X$. For a simplex $\widetilde{\sigma}$ of $\widetilde{B}_{i}$, we denote by $\sigma$ its image $f_{i}(\widetilde{\sigma})$ in $X$. Let $\widetilde{S}_{i}=S_{i}\left(O, \widetilde{B}_{i}\right)$ and let

$$
Z=\left\{(\widetilde{w}, z) \in \widetilde{S}_{i}^{(0)} \times X^{(0)} \mid z \in X_{w} \backslash f_{i}\left(\left(\widetilde{B}_{i}\right)_{\widetilde{w}}\right)\right\} .
$$

Define a relation $\stackrel{e}{\sim}$ on $Z$ as follows:

$$
(\widetilde{w}, z) \stackrel{e}{\sim}\left(\widetilde{w}^{\prime}, z^{\prime}\right) \text { iff }\left(z=z^{\prime} \text { and }\left\langle\widetilde{w}, \widetilde{w}^{\prime}\right\rangle \in \widetilde{B}_{i}^{(1)}\right) .
$$

The transitive closure $\stackrel{\bar{e}}{\sim}$ of the relation $\stackrel{e}{\sim}$ will be further used to define $\widetilde{B}_{i+1}$. The following lemma shows that $\stackrel{\bar{e}}{\sim}$ is not "too far" from $\stackrel{e}{\sim}$.

Lemma 3.5. If $\left(\widetilde{w}_{1}, z\right) \stackrel{e}{\sim}\left(\widetilde{w}_{2}, z\right) \stackrel{e}{\sim}\left(\widetilde{w}_{3}, z\right) \stackrel{e}{\sim}\left(\widetilde{w}_{4}, z\right)$ then there is $(\widetilde{x}, z) \in Z$ with $\left(\widetilde{w}_{1}, z\right) \stackrel{e}{\sim}(\widetilde{x}, z) \stackrel{e}{\sim}\left(\widetilde{w}_{4}, z\right)$. 
Proof. If $\widetilde{w}_{j}=\widetilde{w}_{k}$, for $j \neq k$, or if $\left\langle\widetilde{w}_{1}, \widetilde{w}_{3}\right\rangle \in \widetilde{B}_{i}$, or $\left\langle\widetilde{w}_{1}, \widetilde{w}_{4}\right\rangle \in \widetilde{B}_{i}$, or $\left\langle\widetilde{w}_{2}, \widetilde{w}_{4}\right\rangle \in \widetilde{B}_{i}$, then the assertion trivially holds. Thus further we assume this is not the case. By $\left(P_{i}\right)$ and $\left(Q_{i}\right)$, in $\widetilde{B}_{i-1}$ there are vertices $\widetilde{u}_{1}, \widetilde{u}_{2}, \widetilde{u}_{3}$ with $\widetilde{u}_{j} \sim \widetilde{w}_{j}, \widetilde{w}_{j+1}$.

Claim. For $j \neq k$, we have $\widetilde{u}_{j} \neq \widetilde{u}_{k}$. Furthermore, for $j=1,2, \widetilde{u}_{j} \nsim \widetilde{w}_{j+2}$ and $\widetilde{w}_{j} \nsim \widetilde{u}_{j+2}$.

Proof of Claim. We show only that $\widetilde{u}_{1} \nsim \widetilde{w}_{3}$. Other assertions follow in the same way. By contradiction assume that $\widetilde{u}_{1} \sim \widetilde{w}_{3}$. Observe that $z \nsim u_{1}$, by $\left(R_{i}\right)$ and by the definition of the set $Z$, and that $w_{1} \nsim w_{3}$, by $\left(R_{i}\right)$ and our initial assumptions. Therefore, by $\left(R_{i}\right)$ and by our definition of $Z$ there is a 4 -wheel $\left(w_{2} ; w_{1}, z, w_{3}, u_{1}\right)$ in $X-$ contradiction with 5 -largeness. This finishes the proof of Claim.

By $\left(Q_{i}\right)$, in $\widetilde{B}_{i-1}$ there are vertices $\widetilde{u}_{1}^{\prime}, \widetilde{u}_{2}^{\prime}$ with $\widetilde{u}_{j}^{\prime} \sim \widetilde{u}_{j}, \widetilde{w}_{j+1}, \widetilde{u}_{j+1}$, and $\widetilde{u}_{j}^{\prime} \neq \widetilde{u}_{j}$, by Claim. When $\widetilde{u}_{j} \sim \widetilde{u}_{j+1}$ then $\widetilde{u}_{j}^{\prime}=\widetilde{u}_{j+1}$. We may assume that $\widetilde{u}_{1}^{\prime} \nsim \widetilde{w}_{1}-$ if not then we take $\widetilde{u}_{1}^{\prime}$ instead of $\widetilde{u}_{1}$. Further, we may assume that if $\widetilde{u}_{1}^{\prime} \neq \widetilde{u}_{2}$ then $\widetilde{u}_{1}^{\prime} \nsim \widetilde{w}_{3}$ - if not than we choose $\widetilde{u}_{1}^{\prime}$ instead of $\widetilde{u}_{2}$. By the definition of the set $Z$ and by $\left(R_{i}\right)$, it follows that in $X$ there is a wheel spanned by vertices $w_{2}, z, w_{3}, u_{2}, u_{1}^{\prime}, u_{1}, w_{1}$. Furthermore, if $\widetilde{u}_{2}^{\prime} \neq \widetilde{u}_{2}$ then we may assume that $\widetilde{u}_{2}^{\prime} \nsim \widetilde{w}_{4}-$ otherwise we replace $\widetilde{u}_{3}$ by $\widetilde{u}_{2}^{\prime}$. It may however happen that $\widetilde{u}_{2}^{\prime} \sim \widetilde{w}_{2}$. Then, by the definition of the set $Z$ and by $\left(R_{i}\right)$, in $X$ there is a 5 -wheel $\left(w_{3} ; u_{2}^{\prime}, w_{2}, z, w_{4}, u_{3}\right)$. If $\widetilde{u}_{2}^{\prime} \nsim \widetilde{w}_{2}$ then we have a wheel spanned by vertices $w_{3}, u_{2}^{\prime}, u_{2}, w_{2}, z, w_{4}, u_{3}$. Since $u_{2} \sim u_{2}^{\prime}$, in any case we obtain in $X$ a dwheel $d W$ with the boundary length at most 8 . Therefore, by 8-location, there is a vertex $y$ with $d W \subseteq B_{1}(y, X)$. By $\left(R_{i}\right)$ applied to the vertex $\widetilde{u}_{2}$ there is a vertex $\widetilde{y} \sim \widetilde{u}_{2}$, with $f_{i}(\widetilde{y})=y$. Again by $\left(R_{i}\right)$, we have that all the vertices $\widetilde{w}_{1}, \widetilde{w}_{2}, \widetilde{w}_{3}, \widetilde{w}_{4}, \widetilde{u}_{1}, \widetilde{u}_{2}, \widetilde{u}_{3}, \widetilde{u}_{1}^{\prime}, \widetilde{u}_{2}^{\prime}$ are adjacent to $\widetilde{y}$ in $\widetilde{B}_{i}$. Hence, to prove the lemma it is enough to show that $(\widetilde{y}, z) \in Z$. If not then, by $\left(R_{i}\right)$, there is $\widetilde{z} \in \widetilde{B}_{i}$ such that $\langle\widetilde{z}, \widetilde{y}\rangle \in \widetilde{B}_{i}$. By $\left(R_{i}\right)$, we have then that $\left\langle\widetilde{z}, \widetilde{w}_{1}\right\rangle \in \widetilde{B}_{i}$, which is a contradiction, since $\left(\widetilde{w}_{1}, z\right) \in Z$. This proves the lemma.

Observe that, by Lemma 3.5. if $(\widetilde{u}, z) \stackrel{\bar{e}}{\sim}(\widetilde{w}, z)$ then there is a vertex $\widetilde{y} \in \widetilde{S}_{i}$ with $(\widetilde{y}, z) \in Z$ and $\langle\widetilde{y}, \widetilde{u}\rangle,\langle\widetilde{y}, \widetilde{w}\rangle \in \widetilde{B}_{i}$.

We define the flag simplicial complex $\widetilde{B}_{i+1}$ in the following way. Its 0 -skeleton is by definition the set $\widetilde{B}_{i+1}^{(0)}=B_{i}^{(0)} \cup(Z / \stackrel{\bar{e}}{\sim})$. Now we define the 1 -skeleton $\widetilde{B}_{i+1}^{(1)}$ of $\widetilde{B}_{i+1}$ as follows. Edges between vertices of $\widetilde{B}_{i}$ are the same as in $\widetilde{B}_{i}$. Moreover, for every $\widetilde{w} \in \widetilde{S}_{i}{ }^{(0)}$, there are edges joining $\widetilde{w}$ with $[\widetilde{w}, z] \in Z / \stackrel{\bar{e}}{\sim}$ (here $[\widetilde{w}, z]$ denotes the equivalence class of $(\widetilde{w}, z) \in Z)$ and the edges joining $[\widetilde{w}, z]$ with $\left[\widetilde{w}, z^{\prime}\right]$, for $\left\langle z, z^{\prime}\right\rangle \in X$. Having defined $\widetilde{B}_{i+1}^{(1)}$ the higher dimensional skeleta are determined by the flagness property.

Definition of the map $f_{i+1}: \widetilde{B}_{i+1}^{(0)} \rightarrow X$ is clear: $\left.f_{i+1}\right|_{\widetilde{B}_{i}}=f_{i}$ and $f_{i+1}([\widetilde{w}, z])=z$. We show that it can be simplicially extended. It is enough to do it for simplices in $\widetilde{B}_{i+1} \backslash \widetilde{B}_{i-1}$. Let $\widetilde{\sigma}=\left\langle\left[\widetilde{w}_{1}, z_{1}\right], \ldots,\left[\widetilde{w}_{l}, z_{l}\right], \widetilde{w}_{1}^{\prime}, \ldots\right.$, $\left.\widetilde{w}_{m}^{\prime}\right\rangle \in \widetilde{B}_{i+1}$ be a simplex. Then, by definition of edges in $\widetilde{B}_{i+1}$, we have that $\left\langle z_{p}, z_{q}\right\rangle \in X$ and $\left\langle z_{r}, w_{s}^{\prime}\right\rangle \in X$, for $p, q, r \in\{1,2, \ldots, l\}$ and $s \in\{1,2, \ldots, m\}$. Since $f_{i+1}\left(\left[\widetilde{w}_{p}, z_{p}\right]\right)=z_{p}, f_{i+1}\left(\widetilde{w}_{s}^{\prime}\right)=w_{s}^{\prime}$ and since $f_{i}$ was simplicial, it follows that $\left\langle\left\{f_{i+1}(\widetilde{w}) \mid \widetilde{w} \in \widetilde{\sigma}\right\}\right\rangle \in X$. Hence, by the simplicial extension, we can define the $\operatorname{map} f_{i+1}: \widetilde{B}_{i+1} \rightarrow X$.

Now we check that $\widetilde{B}_{i+1}$ and $f_{i+1}$ satisfy conditions $\left(P_{i+1}\right),\left(Q_{i+1}\right)$ and $\left(R_{i+1}\right)$. The proof of the conditions $\left(P_{i+1}\right)$ and $\left(R_{i+1}\right)$ is exactly the same as the one in the proof of Theorem 4.5 in Osa13 (even up to the notations; see page 12 there).

Condition $\left(Q_{i+1}\right)$. By the condition $\left(Q_{i}\right)$ it is enough to verify the triangle condition $(\mathrm{T})$ and the vertex condition (V) from Definition 3.1 only for, respectively, edges and vertices in $\widetilde{S}_{i+1}$. By the definition of edges in $\widetilde{S}_{i+1}$ it is clear that $\left(\widetilde{B}_{i+1}\right)_{e} \cap \widetilde{B}_{i}$ is non-empty, for an edge $e \in \widetilde{S}_{i+1}$. Therefore, the condition $(\mathrm{T})$ is satisfied. The vertex condition $(\mathrm{V})$, for a vertex $[\widetilde{w}, z] \in \widetilde{S}_{i+1}$ follows immediately from the definition of edges in $\widetilde{B}_{i+1}$, and from Lemma 3.5

Having established conditions $\left(P_{i+1}\right),\left(Q_{i+1}\right)$ and $\left(R_{i+1}\right)$ we are able to construct a complex $\widetilde{X}=\bigcup_{i=1}^{\infty} \widetilde{B}_{i}$ and a map $f=\bigcup_{i=1}^{\infty} f_{i}: \widetilde{X} \rightarrow X$ with the following properties. The complex $\widetilde{X}$ satisfies the property $S D_{n}^{\prime}(O)$ for every $n$ and the map $f$ is a covering map. Thus, by Proposition 2.1, the cover $\widetilde{X}$ is 8-located and locally 5 -large. From Proposition 3.1 it follows that $\widetilde{X}$ is simply connected and therefore $\widetilde{X}$ is the universal cover of $X$. Since the vertex $O$ was chosen arbitrarily in our construction and since the universal cover of $X$ is 
unique it follows that $\widetilde{X}$ satisfies the property $S D_{n}^{\prime}(O)$ for every vertex $O$ and for every natural number $n$. This finishes the proof of the theorem.

\section{5/6*-TRIANGULATIONS ARE 8-LOCATED}

The goal of this section is to show that $5 / 6^{*}$-triangulations are 8 -located locally 5 -large simplicial complexes, i.e. to prove Theorem B from Introduction.

4.1. $5 / 6^{*}$-triangulations of a 2 -sphere. A link of a vertex in a $5 / 6^{*}$-triangulation of a 3 -manifold is a $5 / 6^{*}$-triangulation of a 2-sphere, that is, each vertex has degree (the number of edges adjacent to) 5 or 6 and no two vertices of degree 5 are connected by an edge. A soccer tiling of a 2-sphere is the cellulation dual to a $5 / 6^{*}$-triangulation of a 2 -sphere — see EMM03. Its cells - pentagons and hexagons — correspond to vertices of the triangulation, its vertices correspond to triangles. A soccer diagram is a subcomplex of a soccer tiling, which is homeomorphic to a disc. A path in the boundary of a soccer diagram which is contained in a single cell is called an exposed path.

4.2. 8-location. Throughout this subsection we assume that $X$ is a $5 / 6^{*}$-triangulation of a $3-$ manifold.

Lemma 4.1. $X$ is locally 5 -large and, for $k=5,6$, every $k$-wheel $W$ in $X$ is contained in a link of a vertex $v \notin W$.

The lemma above follows immediately from Lemma 4.2 below. The latter is an elementary result concerning $5 / 6^{*}$-triangulations of a 2 -sphere. For brevity, in the proof we use results from EMM03, Section $3]$.

Lemma 4.2. Let $Y$ be a $5 / 6^{*}$-triangulation of a 2 -sphere. There are no full 4 -cycles in $Y$, and, for $k=5,6$, every full $k$-cycle in $Y$ is contained in a wheel.

Proof. Let $k \in\{4,5,6\}$ and assume there is a full $k$-cycle $c=\left(c_{1}, c_{2}, \ldots, c_{k}\right)$ in $Y$. In the soccer tiling $Y^{\prime}$ corresponding to $Y$, we have then the chain of cells $c_{1}, c_{2}, \ldots, c_{k}$, with $c_{i}$ and $c_{i+1}$ sharing an edge (here and further we use the convention $c_{k+j}=c_{j}$ ). By $c$ we denote the subcomplex of $Y^{\prime}$ being the union of $c_{1}, c_{2}, \ldots, c_{k}$. Observe that if, for $k-1 \geqslant j \geqslant 2, c_{i}$ and $c_{i+j}$ have an edge in common then $c$ (in $Y$ ) is not full, thus further we assume this is not the case. Consider now the two boundary paths $\alpha_{\text {in }}$ and $\alpha_{\text {out }}$ of $c$ in $Y^{\prime}$ - see Figure 4 . Since $k \leqslant 6$ and cells are pentagons or hexagons, the sum of lengths $\left|\alpha_{\text {in }}\right|+\left|\alpha_{\text {out }}\right|$ is at most $6 \cdot 4=24$. Therefore, without loss of generality we assume that $\left|\alpha_{i n}\right| \leqslant 12$. Consider a soccer diagram
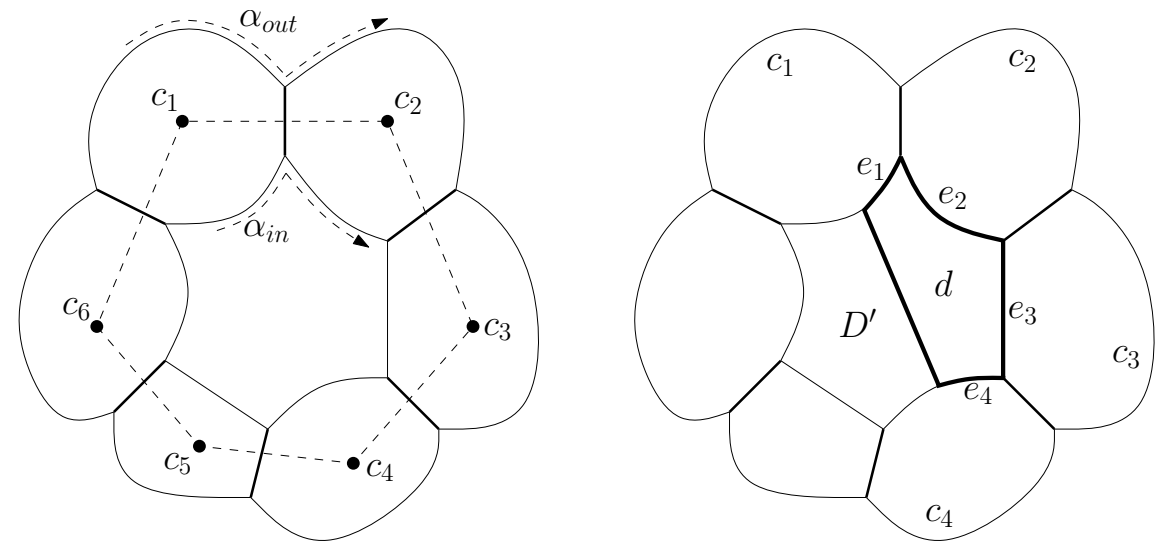

Figure 4. Proof of Lemma 4.2 for $k=6$ : the cycle $c$ and the paths $\alpha_{\text {in }}, \alpha_{\text {out }}$ (left); the exposed path in $D$ (right).

$D$ with boundary $\alpha_{i n}$ that has at most 6 pentagons — such a diagram always exists by EMM03, Remark 2.9]. By EMM03, Theorem 3.10], either $D$ contains an exposed path of length 4 , or $D$ is one of the two diagrams in [EMM03, Figure 2]. It is easy to see that the latter is impossible. Therefore, for the rest of the proof we suppose that there is an exposed path of length 4 in $D$. This is impossible when $k=4$, hence further we consider $k=5,6$. If, for some $i \in\{1,2, \ldots, k\}$, the cell $c_{i}$ belongs to $D$ (i.e. $D$ is "outside $\alpha_{i n}$ " in Figure 4 then $c_{i-1}$ (or $c_{k}$ if $i=1$ ) and $c_{i+1}$ share an edge, contrary to our assumptions. Therefore $D$ 
does not contain any $c_{i}$ (i.e. $D$ is "inside $\alpha_{i n}$ " in Figure 4). Without loss of generality we assume that the exposed path $p$ of length 4 consists of edges $e_{1}, e_{2}, e_{3}, e_{4}$ such that $e_{i} \subseteq c_{i}$. Let $d$ be a cell of $D$ containing $p$. If $D=d$ then the lemma is proved, since then the cycle $c$ (in $Y$ ) is contained in the $k$-wheel $\left(d ; c_{1}, \ldots, c_{k}\right)$ in $Y$. Therefore, further we assume that $D=D^{\prime} \cup d$, where $D^{\prime}$ is a new (nonempty) soccer diagram. Assume that among the cells $c_{i}$ only the cells $c_{1}, c_{2}, \ldots, c_{r}$ intersect $d$, for $4 \leqslant r \leqslant k-1$. Then we obtain a new cycle $c^{\prime}$ of cells: $c_{1}, d, c_{r}, \ldots, c_{k}$, of length strictly smaller than $k$. This corresponds to a full cycle $c^{\prime}$ in $Y$. If $k=5$ then this is impossible as shown above. If $k=6$ then $c^{\prime}$ has to have length 5 and, as we have just proved $c^{\prime}$ is contained in a wheel $\left(d^{\prime} ; c_{1}, d, \ldots\right)$. This means that both $d$ and $d^{\prime}$ are pentagons, which contradicts the definition of the soccer diagram, and thus yields the lemma.

Lemma 4.3. Let $W=\left(v ; c_{1}, c_{2}, \ldots, c_{7}\right)$ be a 7 -wheel in $X$. Then there exist vertices $y \neq z$ such that $\langle v, y, z\rangle \in X$, and, up to renaming cyclically vertices $c_{i}$, the vertex $y$ is adjacent to $c_{1}, c_{2}, c_{3}, c_{4}$, and $z$ is adjacent to $c_{4}, c_{5}, c_{6}, c_{7}, c_{1}$.

Proof. The first part of the proof is the same as in the proof of Lemma 4.2 above: We consider the "cycle" of cells $c_{1}, \ldots, c_{7}$ in the soccer tiling corresponding to $X_{v}$, and we find a path $\alpha_{i n}$ of length at most $7 \cdot 4 / 2=14$. Considering a soccer diagram $D$ with the boundary $\alpha_{i n}$ and with at most 6 pentagons, we conclude that $D$ does not contain any $c_{i}$, and that there is an exposed path of length 4 . It is not possible that $D$ consists of a single cell, so that the cell $d$ containing the exposed path forms a new cycle of less than 7 cells. For the new cycle we apply Lemma 4.2 , to obtain a single "filling" cell $f$. The pair of cells $(d, f)$ corresponds to the pair of required vertices: $(y, z)$ or $(z, y)$.

Proof of Theorem B. Assume that $d W=\left(v_{0}, v_{0}^{\prime}, w ; v_{1}, \ldots, v_{k-2} ; v_{1}^{\prime}, \ldots, v_{l-2}^{\prime}\right)$ is a $(k, l)$-dwheel in $X$ with the boundary length at most 8 . Without loss of generality we assume that $k \geqslant l$. By Lemma 4.1 the only possibilities for the pair $(k, l)$ are: $(5,5),(6,5),(6,6),(7,5)$. By Lemma 4.1, there is a vertex $\bar{v}_{0}^{\prime} \neq v_{0}^{\prime}$ such that $\left(v_{0}^{\prime} ; v_{1}^{\prime}, \ldots, v_{l-2}^{\prime}, w, v_{0}\right) \subseteq X_{\bar{v}_{0}^{\prime}}$.

First we consider the case when $k \leqslant 6$. By Lemma 4.1, there is a vertex $\bar{v}_{0} \neq v_{0}$ such that the wheel $\left(v_{0} ; v_{1}, \ldots, v_{k-2}, w, v_{0}^{\prime}\right) \subseteq X_{\bar{v}_{0}}$. If $\bar{v}_{0}=\bar{v}_{0}^{\prime}$ then $d W \subseteq B_{1}\left(\bar{v}_{0}\right)$ and we are done. Hence further we assume that $\bar{v}_{0} \neq \bar{v}_{0}^{\prime}$. If $v_{1}=v_{1}^{\prime}$ then $\left(v_{1}, \bar{v}_{0}, w, \bar{v}_{0}^{\prime}\right)$ is a 4-cycle in $X_{\left\langle v_{0}, v_{0}^{\prime}\right\rangle}$. By Lemma 4.1 this cycle is not full and thus $\left\langle\bar{v}_{0}, \bar{v}_{0}^{\prime}\right\rangle \in X$. Hence, the vertices $v_{0}, v_{0}^{\prime}, v_{1}, \bar{v}_{0}, \bar{v}_{0}^{\prime}$ span a 4 -simplex - contradiction. If $v_{1} \neq v_{1}^{\prime}$ then, by the definition of a dwheel $d W$, we have $v_{1} \sim v_{1}^{\prime}$ and, by our assumptions, $l=5$. In this case the cycle $\left(v_{1}, \bar{v}_{0}, w, \bar{v}_{0}^{\prime}, v_{1}^{\prime}\right)$ is a 5 -cycle in $X_{\left\langle v_{0}, v_{0}^{\prime}\right\rangle}$. If this cycle is not full then we have a contradiction as before. If it is full then the edge $\left\langle v_{0}, v_{0}^{\prime}\right\rangle$ has degree 5 . However, also the edge $\left\langle v_{0}^{\prime}, \bar{v}_{0}^{\prime}\right\rangle$ has degree 5 contradicting the definition of the $5 / 6^{*}$-triangulation.

It remains to treat the case $(k, l)=(7,5)$. Observe that in this case we have $v_{1}=v_{1}^{\prime}$. Let $y \neq z$ be vertices as in Lemma 4.3 for the 7 -wheel $\left(v_{0} ; v_{1}, \ldots, v_{k-2}, w, v_{0}^{\prime}\right)$. Either $v_{1}, v_{0}^{\prime}, w$ are all adjacent to $y$ or to $z$, or, without loss of generality, we assume that $\left\langle z, w, v_{0}^{\prime}\right\rangle \in X$, and $\left\langle y, v_{1}, v_{0}^{\prime}\right\rangle \in X$. In the former case we proceed as above for $k \leqslant 6$. Thus further we assume that $\langle y, w\rangle,\left\langle z, v_{1}\right\rangle \notin X$. Then there is a 5 -cycle $\left(y, z, w, \bar{v}_{0}^{\prime}, v_{1}\right)$ in the link of the edge $\left\langle v_{0}, v_{0}^{\prime}\right\rangle$. If this cycle is not full then we obtain a simplex of dimension above 3 in $X-$ contradiction. Therefore the cycle is full and hence the degree of $\left\langle v_{0}, v_{0}^{\prime}\right\rangle$ is 5 . This means however that there are two degree 5 edges in the triangle $\left\langle v_{0}, v_{0}^{\prime}, \bar{v}_{0}^{\prime}\right\rangle$, contradicting the definition of the $5 / 6^{*}$-triangulation.

\section{Further APPLiCATIONS AND FinAl REMARKS}

5.1. Versions of $m$-location. There is another, "more natural", version of $m$-location, that originated in fact our studies: A flag (now, not necessarily 5 -large) simplicial complex is $m$-located if every homotopically trivial loop of length at most $m$ has a filling diagram with at most one internal vertex (see eg. JŚ06. Osa13 for basics on filling diagrams). The new property does not imply the one considered in this paper, neither vice versa. The following analogue of Theorem A holds.

Theorem. Let $X$ be a simply connected 8-located flag simplicial complex. Then the 1-skeleton of X, equipped with the standard path metric, is Gromov hyperbolic.

A more general scheme for local conditions implying Gromov hyperbolicity relies on the requirement that "short loops allow small filling diagrams". This leads to particular instances of the local-to-global principle for the linear Dehn function.

In the next subsections one may usually consider both versions of " $m$-location" when it is mentioned. 
5.2. Further applications. The notion of 8-location can be applied not only in the case of (closed) manifolds or manifolds with boundaries. Having, a $5 / 6^{*}$-triangulation of a 3 -manifold one can construct a branched cover that will still be an 8-located locally 5-large complex. There are also various amalgamation procedures that can be applied to obtain "singular" spaces beginning with manifolds. Further, it is possible to construct 8-located pseudomanifolds using constructions from JŚ06 and similar (cf. e.g. Osa13 for further references). Objects obtained in this way may be asymptotically different from the existing ones compare the next subsection.

Clearly, 8-location applies to higher dimensional manifolds as well. Nevertheless, it is an interesting question, whether for 4-manifolds (and in higher dimensions) one may formulate conditions on a triangulation in the spirit of Thurston's condition, implying 8-location, and thus hyperbolicity.

5.3. Relations to other combinatorial nonpositive curvature conditions. There are various notions of "combinatorial nonpositive curvature" appearing in the literature - see e.g. $\mathrm{BCC}^{+} 13$ Osa13 for more details on this. In particular, for $k \geqslant 6$, a $k$-systolic complex JŚn 06 is a simply connected locally $k$-large simplicial complex. In Osa13 the notion of a weakly systolic complex, being a generalization of $k$-systolicity, has been introduced. The following immediate corollary of Theorem A provides a local condition on a weakly systolic complex implying its hyperbolicity. The new condition is more general than the well studied conditions for hyperbolicity: local 7-largeness for $k$-systolic complexes (see [JŚ06, Section 2]), and $S D_{2}^{*}(7)$ for weakly systolic complexes (see Osa13, Section 7]).

Corollary 1. The following condition implies hyperbolicity of a weakly systolic complex X: For every vertex $v$ such that $X_{v}$ is not 6-large (respectively, is 6-large but not 7-large), every its neighbor $w$ has an 8-large (respectively, 7-large) link $X_{w}$.

Note however, that (weakly) systolic complexes do not fit well the world of manifolds of dimension above 2 - e.g. there are no $k$-systolic triangulations of such manifolds [JŚ06. More strikingly, groups acting geometrically on systolic complexes are far from acting in the same way on aspherical manifolds - see e.g. Osa07]. Thus the 8-location is a (asymptotically) far going generalization of 7-systolicity, that allows still to obtain strong results on the structure of the complex - as e.g. in the description of combinatorial balls provided within the proof of Theorem 3.4

5.4. Beyond 8-location. In the current paper we focus on 8-located complexes. Nevertheless, the 7location seems to be an interesting nonpositive-curvature-like property, as argued below. It is not so with 6-location - there exist 6-located locally 5-large triangulations of the 2-sphere.

Note that all 6-systolic complexes are 7-located. In particular, the 7-location does not imply hyperbolicity: The tiling of the Euclidean plane by equilateral triangles is 7 -located. However, with much more effort than for the 8-location, one can prove a local-to-global result similar to Theorem 3.4 also for 7-located complexes. We believe that there are other nonpositive-curvature-like properties satisfied by such complexes (cf. e.g. [JŚ06 Osa13]). This, together with the fact that 7-location goes far beyond systolicity, make us believe that this notion deserves further studies.

\section{REFERENCES}

[BCC $\left.{ }^{+} 13\right]$ B. Brešar, J. Chalopin, V. Chepoi, T. Gologranc, and D. Osajda, Bucolic complexes, Adv. Math. 243 (2013), 127-167, DOI 10.1016/j.aim.2013.04.009. MR3062742

[EMM03] M. Elder, J. McCammond, and J. Meier, Combinatorial conditions that imply word-hyperbolicity for 3-manifolds, Topology 42 (2003), no. 6, 1241-1259, DOI 10.1016/S0040-9383(02)00100-3. MR1981356 (2004f:20078)

[JŚ06] T. Januszkiewicz and J. Świątkowski, Simplicial nonpositive curvature, Publ. Math. Inst. Hautes Études Sci. 104 (2006), 1-85, DOI 10.1007/s10240-006-0038-5. MR2264834 (2007j:53044)

[Osa07] D. Osajda, Connectedness at infinity of systolic complexes and groups, Groups Geom. Dyn. 1 (2007), no. 2, 183-203, DOI 10.4171/GGD/9. MR2319456 (2008e:20064)

[Osa13] __ A combinatorial non-positive curvature I: weak systolicity (2013), preprint, available at arXiv:1305.4661

[Pap95] P. Papasoglu, Strongly geodesically automatic groups are hyperbolic, Invent. Math. 121 (1995), no. 2, 323-334, DOI 10.1007/BF01884301. MR1346209 (96h:20073)

Instytut Matematyczny, Uniwersytet WrocŁawski, Pl. Grunwaldzki 2/4, 50-384 Wroceaw, Poland

Universität Wien, Fakultät für Mathematik, Oskar-Morgenstern-Platz 1, 1090 Wien, Austria.

E-mail address: dosaj@math.uni.wroc.pl 